\title{
ASYMPTOTIC DECOMPOSITION OF LINEAR SINGULARLY PERTURBED MULTISCALE SYSTEMS
}

\author{
I. CHEREVKO AND O. OSYPOVA
}

Received 13 April, 2015

\begin{abstract}
We study a scheme which decomposes a singularly perturbed linear system with several small parameters into a block-diagonal form. The algorithm of the scheme contains $k$ steps of a successive splitting based on the integral manifold of quick and slow variables method. The splitting substitution is defined constructively in the form of expansions by powers of small parameters.
\end{abstract}

2010 Mathematics Subject Classification: 34A30; 34C45; 34D15; 34E15; 37D10

Keywords: linear singularly perturbed systems, decomposition, asymptotic decomposition, integral manifolds

\section{INTRODUCTION}

The theory of singularly perturbed systems of differential equations has been intensively developed recently, and its methods have been widely used for the study of various problems of science and technology. Singularly perturbed models describe dynamic processes in physics, theory of automatic control, hydroscopic systems, economical and biological processes [2,3]. Singularly perturbed systems have boundary layer and stiffness property in numerical calculations. In this connection the main results for high-dimensional systems with fast and slow variables boil down to the construction of asymptotic expansions of the initial and boundary problems. Let us note the surveys $[6,8,13]$, where a great amount of works dedicated to this subject were analyzed.

For qualitative studies of entire systems it is expedient to apply decomposition algorithms. Effective methods of decomposition of singularly perturbed systems, which are based on the ideas of the integral manifold method, were developed in the works [11,12]. For linear singularly perturbed systems such an approach allows us to make the decomposition of an input system to the independent fast and slow subsystems [7,10]. Linear non-autonomous three-time and n-time scale systems were studied in $[1,4,9]$. 
The aim of this paper is to justify the decomposition method of a linear singularly perturbed system with several small parameters to the collection of independent subsystems. Such an approach is effective if we can find the coefficients of the splitting transformation. The suggested approach combines both asymptotic and qualitative methods of analysis. The splitting transformation is constructed with an arbitrary precision as asymptotic expansions in a series of small parameters.

\section{THE SCHEME OF DECOMPOSITION}

Consider a linear singularly perturbed system given by

$$
\prod_{j=0}^{i} \varepsilon_{j} \dot{x}_{i}=\sum_{j=0}^{k} A_{i j} x_{j}, i=\overline{0, k},
$$

where $t \in \mathbb{R}, x_{i} \in \mathbb{R}^{n_{i}}, A_{i j}=A_{i j}(t), i, j=\overline{0, k}$, are $n_{i} \times n_{j}$ matrices, $\varepsilon_{0}=1$, $\varepsilon_{1}, \varepsilon_{2}, \ldots, \varepsilon_{k}$ are small positive parameters.

Let the following conditions be true:

1) matrices $A_{i j}(t), i, j=\overline{0, k}$, are uniformly bounded in $t \in \mathbb{R}$,

2) eigenvalues of the matrix $A_{k k}(t)$ satisfy the inequality

$$
\operatorname{Re} \lambda_{j}\left(A_{k k}\right) \leq-2 \beta<0, j=\overline{1, n_{k}} .
$$

The decomposition of system (2.1) will be performed in $k$ steps. At the first step the substitution is made as follows

$$
\left\{\begin{array}{l}
x_{i}=y_{i}^{1}+\prod_{j=i+1}^{k} \varepsilon_{j} H_{i}^{1} y_{k}^{1}, i=\overline{0, k-1} \\
x_{k}=y_{k}^{1}+\sum_{j=0}^{k-1} P_{j}^{1} x_{j}
\end{array}\right.
$$

where $H_{i}^{1}, P_{i}^{1}, i=\overline{0, k-1}$ are matrix functions of appropriate dimensions.

If $H_{i}{ }^{1}, P_{i}^{1}, i=\overline{0, k-1}$ are bounded solutions of the systems

$$
\begin{gathered}
\prod_{j=0}^{k} \varepsilon_{j} \dot{P}_{i}^{1}=A_{k i}+A_{k k} P_{i}^{1}-\sum_{j=0}^{k-1} \prod_{m=j+1}^{k} \varepsilon_{m} P_{j}^{1}\left(A_{j i}+A_{j k} P_{i}^{1}\right), \\
\prod_{j=0}^{k} \varepsilon_{j} \dot{H}_{i}^{1}=A_{i k}-H_{i}^{1}\left(A_{k k}-\sum_{j=0}^{k-1} \prod_{m=j+1}^{k} \varepsilon_{m} P_{j}^{1} A_{j k}\right)+ \\
+\sum_{j=0}^{k-1} \prod_{m=j+1}^{k} \varepsilon_{m}\left(A_{i j}+A_{i k} P_{j}^{1}\right) H_{j}^{1},
\end{gathered}
$$


then system (2.1) has the form

$$
\begin{gathered}
\prod_{j=0}^{i} \varepsilon_{j} \dot{y}_{i}^{1}=\sum_{j=0}^{k-1} B_{i j}^{1} y_{j}^{1}, \quad i=\overline{0, k-1} \\
\prod_{j=0}^{k} \varepsilon_{j} \dot{y}_{k}^{1}=B_{k k}^{1} y_{k}^{1}
\end{gathered}
$$

where $B_{i j}^{1}=A_{i j}+A_{i k} P_{j}^{1}, i, j=\overline{0, k-1}, B_{k k}^{1}=A_{k k}-\sum_{j=0}^{k-1} \prod_{m=j+1}^{k} \varepsilon_{m} P_{j}^{1} A_{j k}$.

To perform the second step of decomposition, we make such assumption:

3) eigenvalues of $B_{k-1, k-1}^{1}$ satisfy the inequality

$$
\operatorname{Re} \lambda_{i}\left(B_{k-1, k-1}^{1}\right) \leq-2 \gamma<0 .
$$

Then by making the substitution

$$
\left\{\begin{array}{l}
y_{i}^{1}=y_{i}^{2}+\prod_{j=i+1}^{k-1} \varepsilon_{j} H_{i}^{2} y_{k-1}^{2}, i=\overline{0, k-2}, \\
y_{k-1}^{1}=y_{k-1}^{2}+\sum_{j=0}^{k-2} P_{j}^{2} y_{j}^{1}
\end{array}\right.
$$

two independent subsystems are obtained from system (2.4a)

$$
\begin{gathered}
\prod_{j=0}^{i} \varepsilon_{j} \dot{y}_{i}^{2}=\sum_{j=0}^{k-2} B_{i j}^{2} y_{j}^{2}, \quad i=\overline{0, k-2} \\
\prod_{j=0}^{k-1} \varepsilon_{j} \dot{y}_{k-1}^{2}=B_{k-1, k-1}^{2} y_{k-1}^{2},
\end{gathered}
$$

where $B_{i j}^{2}=B_{i j}^{1}+B_{i, k-1}^{1} P_{j}^{2}, i, j=\overline{0, k-2}, B_{k-1, k-1}^{2}=B_{k-1, k-1}^{1}-$ $-\sum_{j=0}^{k-2} \prod_{m=j+1}^{k-1} \varepsilon_{m} P_{j}^{2} B_{j k}^{1}$.

Matrices $P_{i}^{2}, H_{i}^{2}, i=\overline{0, k-2}$, are uniformly bounded solutions of the systems

$$
\begin{gathered}
\prod_{j=0}^{k-1} \varepsilon_{j} \dot{P}_{i, 2}=B_{k-1, i}^{1}+B_{k-1, k-1}^{1} P_{i}^{2}-\sum_{j=0}^{k-2} \prod_{m=j+1}^{k-1} \varepsilon_{m} P_{j}^{2}\left(B_{j i}^{1}+B_{j, k-1}^{1} P_{i, 2}\right), \\
\prod_{j=0}^{k-1} \varepsilon_{j} \dot{H}_{i}^{2}=B_{i, k-1}^{1}-H_{i}^{2}\left(B_{k-1, k-1}^{1}-\sum_{j=0}^{k-2} \prod_{m=j+1}^{k-1} \varepsilon_{m} P_{j}^{2} B_{j, k-1}^{1}\right)+
\end{gathered}
$$




$$
+\sum_{m=0}^{k-2} \prod_{n=m+1}^{k-1} \varepsilon_{n}\left(B_{i m}^{1}+B_{i, k-1}^{1} P_{m}^{2}\right) H_{m}^{2}, \quad i=\overline{0, k-2} .
$$

At the step $k$ by making the substitution

$$
\left\{\begin{array}{l}
y_{0}^{k-1}=y_{0}^{k}+\varepsilon_{1} H_{0}^{k} y_{1}^{k} \\
y_{1}^{k-1}=y_{1}^{k}+P_{0}^{k} y_{0}^{k-1}
\end{array}\right.
$$

we obtain the block-diagonal system

$$
\left\{\begin{array}{l}
\dot{y}_{0}^{k}=B_{00}^{k} y_{0}^{k}, \\
\prod_{j=0}^{i} \varepsilon_{j} \dot{y}_{i}^{k-i+1}=B_{i i}^{k-i+1} y_{i}^{k-i+1}, i=\overline{1, k},
\end{array}\right.
$$

where $B_{00}^{k}=B_{00}^{k-1}+B_{01}^{k-1} P_{0}^{k}, B_{11}^{k}=B_{11}^{k-1}-\varepsilon_{1} P_{0}^{k} B_{01}^{k-1}$.

Here the matrices $H_{0}^{k}, P_{0}^{k}$ of appropriate dimensions satisfy the equations

$$
\begin{aligned}
\varepsilon_{1} \dot{P}_{0}^{k} & =B_{10}^{k-1}+B_{11}^{k-1} P_{0}^{k}-\varepsilon_{1} P_{0}^{k}\left(B_{00}^{k-1}+B_{02}^{k-1} P_{0}^{k}\right), \\
\varepsilon_{1} \dot{H}_{0}^{k} & =B_{01}^{k-1}-H_{0}^{k}\left(B_{11}^{k-1}-\varepsilon_{1} P_{0}^{k} B_{01}^{k-1}\right)+ \\
& +\varepsilon_{1}\left(B_{00}^{k-1}+B_{02}^{k-1} P_{0}^{k}\right) H_{0}^{k} .
\end{aligned}
$$

At each step of decomposition we assume that

$3_{i}$ ) eigenvalues of matrices $B_{i i}^{k-i+1}, i=\overline{1, k-1}$, satisfy the inequality

$$
\operatorname{Re} \lambda_{j}\left(B_{i i}^{k-i+1}\right) \leq-2 \gamma<0 .
$$

For the transformation of system (2.1) to block-diagonal form (2.9) the existence of the corresponding solutions of systems (2.3a)-(2.3b), (2.7), .., (2.10) is necessary. Then, we have the following lemma.

Lemma 1. Let conditions 1)-2) be true. Then there exists $\varepsilon_{k}^{\star}>0$ such that for any $\varepsilon_{k} \in\left(0, \varepsilon_{k}^{\star}\right)$ system (2.3a)-(2.3b) has a unique bounded solution for $t \in \mathbb{R}$.

Proof. Write the subsystem (2.3a) in the equivalent form

$$
\begin{array}{r}
P_{i}(t, s, \varepsilon)=\frac{1}{\prod_{j=0}^{k} \varepsilon_{j}-\infty} \int_{j=0}^{t} Q(t, s, \varepsilon)\left(A_{k i}(s, \varepsilon)-\sum_{j=j+1}^{k-1} \prod_{m}^{k} \varepsilon_{m} P_{j}(s, \varepsilon)\left(A_{i j}(s, \varepsilon)+\right.\right. \\
\left.\left.+A_{j k}(s, \varepsilon) P_{i}(s, \varepsilon)\right)\right) d s, \quad i=\overline{0, k-1}, \quad
\end{array}
$$

where $Q(t, s, \varepsilon)$ is the fundamental matrix of the equation

$$
\prod_{j=0}^{k} \varepsilon_{j} \dot{x}_{k}=A_{k k} x_{k}
$$


which by 1)-2) satisfies the estimation

$$
\|Q(t, s, \varepsilon)\| \leq K e^{-\frac{3 \beta}{2 \prod_{j=0}^{k} \varepsilon_{j}}(t-s)},
$$

for some $K>0$ and any $-\infty<s \leq t<\infty$.

We shall seek a bounded solution of system (2.11) on the entire real axis by the method of successive approximations

$$
\begin{gathered}
P_{i}^{n+1}=\frac{1}{\prod_{j=0}^{k} \varepsilon_{j}} \int_{-\infty}^{t} Q\left(A_{k i}-\sum_{j=0}^{k-1} \prod_{m=j+1}^{k} \varepsilon_{m} P_{j}^{n}\left(A_{j i}+A_{j k} P_{i}^{n}\right)\right) d s, \\
i=\overline{0, k-1}, n=0,1,2, \ldots,
\end{gathered}
$$

putting $P_{i}^{0}=0, i=\overline{0, k-1}$.

Using inequality (2.13) and conditions 1)-2) in the way analogous to [5] it is easy to show that there exists $\varepsilon_{k}^{\star}>0$ such that for all $\varepsilon_{k} \in\left(0, \varepsilon_{k}^{\star}\right)$ sequences $P_{i}^{n}(t, \varepsilon), i=$ $\overline{0, k-1}$, are uniformly convergent as $n \rightarrow \infty$ and bounded for $t \in \mathbb{R}$.

Put $P_{i}(t, \varepsilon)=\lim _{n \rightarrow \infty} P_{i}^{n}(t, \varepsilon), i=\overline{0, k-1}$.

Considering the correlation for matrices $B_{i j}, i, j=\overline{0, k}$ we shall rewrite (2.3b) in the form

$$
\prod_{j=0}^{k} \varepsilon_{j} \dot{H}_{i}^{1}=A_{i k}+\sum_{j=0}^{k-1} \prod_{m=j+1}^{k} \varepsilon_{m} B_{i j} H_{j}^{1}-H_{i}^{1} B_{k k}, i=\overline{0, k-1} .
$$

Denote by $Q_{H_{i}}(t, s, \varepsilon)$ the fundamental matrices of the equations

$$
\prod_{j=0}^{i} \varepsilon_{j} \dot{x}_{i}=B_{i j}(t, \varepsilon) x_{i}, \quad i=\overline{0, k-1}
$$

and by $\bar{Q}(t, s, \varepsilon)$ the fundamental matrix of the equation

$$
\prod_{i=0}^{k} \varepsilon_{i} \dot{y}_{k}^{1}=\left(A_{k k}-\sum_{i=0}^{k-1} \prod_{j=i+1}^{k} \varepsilon_{j} P_{i}^{1} A_{i k}\right) y_{k}^{1} .
$$

We can equivalently rewrite system (2.15) as follows

$$
H_{i}^{1}=-\frac{1}{\prod_{j=1}^{k} \varepsilon_{j}} \int_{-\infty}^{t} \bar{Q}\left(A_{i k}+\sum_{\substack{j=0, m=j+1 \\ j \neq i}}^{k-1} \prod_{\substack{k \\ j}}^{k} \varepsilon_{i j} H_{j}^{1}\right) Q_{H_{i}} d s, i=\overline{0, k-1}
$$


If conditions 1)-2) are satisfied and $\varepsilon_{k}$ are sufficiently small then the fundamental matrix $\bar{Q}(t, s, \varepsilon)$ satisfies the estimation [5]

$$
|\bar{Q}(t, s, \varepsilon)| \leq K e^{-\frac{\beta}{\prod_{i=0}^{k} \varepsilon_{i}}(t-s)} .
$$

Using inequality (2.18) we can prove the existence of a bounded on the entire real axis solution of system (2.17) in a similar way as for (2.11).

The lemma is proved.

Similar statements for systems $(2.7), \ldots,(2.10)$ are true.

Let us obtain now a general decomposing transformation.

Theorem 1. Let conditions 1)-3) be true. Then for sufficiently small parameters $\varepsilon_{i}, i=\overline{1, k}$, there exists a nonsingular substitution

$$
\left(x_{0}, x_{1}, x_{2}, \ldots, x_{k}\right)^{T}=\Phi\left(y_{0}^{k}, y_{1}^{k}, y_{2}^{k-1}, \ldots, y_{k}^{1}\right)^{T},
$$

which transforms system (2.1) to $(k+1)$ independent subsystems (2.9).

Proof. The structure of elements of decomposition transformation

$$
\Phi[i, j]=\left\{\begin{array}{l}
R_{j}^{k-j}+\sum_{m=0}^{j-1} \prod_{n=m+1}^{j} \varepsilon_{n} R_{m}^{k-j+1} H_{m}^{k-j+1}, \quad(i \geq j) \wedge(i>1), \\
R_{i}^{k-i}=E, R_{m}^{k-i+1}=P_{m}^{k-i+1}, \\
R_{m}^{n+1}=R_{m}^{n}+R_{k-n}^{n} P_{m}^{n+1}, \\
\prod_{m=i+1}^{j} \varepsilon_{m} H_{i}^{k+1-j} y_{j}^{k+1-j}, \quad(i<j) \wedge(j>1) \\
E, i=j=0 \\
\varepsilon_{1} H_{0}^{k}, i=0, j=1, \\
P_{0}^{k}, i=1, j=0 \\
E+\varepsilon_{1} P_{0}^{k} H_{0}^{k}, i=j=1
\end{array}\right.
$$

follows from the representation of the substitution at each step of the decomposition. Let us show that this transformation is nonsingular.

From (2.4a), (2.4b) we obtain

$$
\left\{\begin{array}{l}
y_{i}^{1}=x_{i}-\prod_{j=i+1}^{k} \varepsilon_{j} H_{i}^{1} y_{k}^{1}, \quad i=\overline{0, k-1} \\
y_{k}^{1}=x_{k}-\sum_{j=0}^{k-1} P_{j}^{1} x_{j}
\end{array}\right.
$$


Now by making the next substitution we obtain

$$
\begin{aligned}
& y_{0}^{k}=\sum_{j=0}^{k} D_{0 j}^{k+1} x_{j}, \\
& y_{i}^{k+1-i}=\sum_{j=0}^{k} D_{i j}^{k+1-i} x_{j},
\end{aligned}
$$

where for $n=\overline{0, k-1}, j=\overline{0, k}$ one has

$$
\begin{aligned}
& D_{k-n, j}^{n+1}=D_{k-n, j}^{n}-\sum_{m=0}^{k-n-1} P_{m}^{n+1} D_{k-n, j}^{n+1}, \\
& D_{i j}^{n+1}=D_{i j}^{n}-\prod_{m=i+1}^{k-n} \varepsilon_{m} H_{i}^{n+1} D_{k-n, j}^{n+1}, i=\overline{0, k-n-1}, \\
& D_{i j}^{0}=0, i \neq j, D_{i i}^{0}=E, \\
& D_{0 j}^{k+1}=\left(E+\varepsilon_{1} H_{0}^{k} P_{0}^{k}\right) D_{0 j}^{k-1}-\varepsilon_{1} H_{0}^{k} D_{1 j}^{k-1} .
\end{aligned}
$$

Therefore, there exists the inverse matrix $\Phi^{-1}[i, j]=D_{i j}^{k+1-i}, i=\overline{0, k}$.

The theorem is proved.

Remark 1 . Condition $3_{i}$ ) is difficult to verify, because matrices $P_{j}^{i}$ and $H_{j}^{i}$ can be found only in the simplest cases. It is easy to make sure that condition $3_{i}$ ) will be verified for small $\varepsilon_{1}, \varepsilon_{2}, \ldots, \varepsilon_{k-1}$, if system (2.1) is of such form

$$
\left\{\begin{array}{l}
\dot{x}_{0}=\sum_{j=0}^{k} A_{0 j} x_{j}, \\
\prod_{j=0}^{i} \varepsilon_{j} \dot{x}_{i}=\sum_{j=0, j \neq i+1}^{k} A_{i j} x_{j}+\varepsilon_{i} A_{i, i+1} x_{i+1}, \quad i=\overline{1, k},
\end{array}\right.
$$

and the eigenvalues of the matrices $A_{i i}, i=\overline{1, k}$, satisfy the inequality

$$
\operatorname{Re} \lambda_{j}\left(A_{i i}\right) \leq-2 \gamma<0 .
$$

\section{ASYMPTOTIC DECOMPOSITION OF THE SPLITTING TRANSFORMATION.}

We can find the exact form for the coefficients of the asymptotic decomposition only in the simplest cases, so we are interested in finding asymptotic decomposition of these coefficients and obtaining corresponding split systems.

Let us make the following important assumption:

4) matrices $A_{i j}(t), i, j=\overline{0, k}, A_{k k}^{-1}(t)$ and their derivatives up to $(n+1)$ order are uniformly bounded in $t \in \mathbb{R}$.

Consider the differential expression

$$
T^{0}\left(u^{0}\right)=\sum_{i=0}^{k-1} A_{k i} x_{i}+A_{k k} u^{0}-\prod_{i=0}^{k} \varepsilon_{i} \frac{d}{d t} u^{0} .
$$


We show that the function $u^{0}\left(t, x_{0}(t), x_{1}(t), \ldots, x_{k-1}(t), \varepsilon_{k}\right)$, which can be represented in the form

$$
u^{0}=\sum_{i=0}^{k-1} \bar{P}_{i}^{1}\left(t, \varepsilon_{k}\right) x_{i}=\sum_{i=0}^{k-1}\left(\sum_{j=0}^{n} \varepsilon_{k}^{j} P_{i, j}^{1}(t)\right) x_{i},
$$

where matrices $P_{i, j}^{1}(t), j=\overline{0, n}, i=\overline{0, k-1}$, and their $(n-j+1)$ derivatives are uniformly bounded, is such that for bounded solutions of system (2.1) one has $T^{0}\left(u^{0}\right)=$ $o\left(\varepsilon_{k}^{n+1}\right)$.

We substitute (3.2) to (3.1) and choose the functions

$$
P_{i, j}^{1}(t), j=\overline{0, n}, i=\overline{0, k-1},
$$

so that in (3.1) all the terms that contain powers of $\varepsilon_{k}$ less than $(n+1)$, are vanishing. For the coefficients in representation (3.2) we obtain

$$
\begin{aligned}
P_{i, 0}^{1}(t) & =-A_{k k}^{-1}(t) A_{k i}(t), \\
P_{i, j}^{1}(t) & =A_{k k}^{-1}(t)\left(\prod_{m=1}^{k-1} \varepsilon_{m} \dot{P}_{i, j-1}^{1}(t)+\sum_{m=0}^{k-1} \prod_{l=m+1}^{k-1} \varepsilon_{l} P_{m, j-1}^{1}(t) A_{m i}(t)+\right. \\
+ & \left.\sum_{m=0}^{k-1} \prod_{l=m+1}^{k-1} \varepsilon_{l} \sum_{l=0}^{j-1} P_{m, l}^{1}(t) A_{m k}(t) P_{i, j-l-1}^{1}(t)\right), \quad j=\overline{1, n} .
\end{aligned}
$$

Observe that 4) implies that $P_{i, j}^{1}(t), j=\overline{0, n}, i=\overline{0, k-1}$, and their partial derivatives up to $(n-j+1)$ order, are bounded. If the functions $P_{i, j}^{1}$ are chosen by formulaes (3.3), then (3.1) has the form

$$
T^{0}\left(u^{0}\right)=\varepsilon_{k}^{n+1}\left(\sum_{i=0}^{k-1} \eta_{i}^{0}\left(t, \varepsilon_{1}, \ldots, \varepsilon_{k-1}\right) x_{i}\right),
$$

where $\eta_{i}^{0}, i=\overline{0, k-1}$, are uniformly bounded functions.

If we make in input system (2.1) the substitution $x_{k}=\sum_{i=0}^{k-1} \bar{P}_{i}^{1} x_{i}+\varepsilon_{k}^{n+1} z$, then for the variables $x_{0}, x_{1}, \ldots, x_{k-1}, z$ we obtain the system

$$
\left\{\begin{array}{l}
\prod_{j=0}^{i} \varepsilon_{j} \dot{x}_{i}=\sum_{j=0}^{k-1}\left(A_{i j}+A_{i k} \bar{P}_{j}^{1}\right) x_{j}+\varepsilon_{k}^{n+1} A_{i k} z, \quad i=\overline{0, k-1} \\
\prod_{j=0}^{k} \varepsilon_{j} \dot{z}=\sum_{j=0}^{k-1} \eta_{j}^{0}\left(t, \varepsilon_{1}, \ldots, \varepsilon_{k-1}\right) x_{j}+A_{k k} z
\end{array}\right.
$$


System (3.4) is a system of type (2.1), for which there exists an integral manifold [12]

$$
z=\sum_{i=0}^{k-1} P_{i, \star}^{1}\left(t, \varepsilon_{k}\right) x_{i}
$$

where $P_{i, \star}^{1}, i=\overline{0, k-1}$ are uniformly bounded functions.

If system (3.4) has integral manifold (3.5), then system (2.1) has the integral manifold

$$
x_{k}=\sum_{i=0}^{k-1}\left(\bar{P}_{i}^{1}+\varepsilon_{k}^{n+1} P_{i, \star}^{1}\right) x_{i}=\sum_{i=0}^{k-1} P_{i}^{1} x_{i}
$$

for which the asymptotic decomposition

$$
x_{k}=\sum_{i=0}^{k-1}\left(\sum_{i=0}^{k-1} \varepsilon_{k}^{j} P_{i, j}^{1}+\varepsilon_{k}^{n+1} P_{i, \star}^{1}\right) x_{i}
$$

is valid.

By making in system (2.1) the substitution $x_{k}=\sum_{i=0}^{k-1} P_{i}{ }^{1} x_{i} y_{k}^{1}$, we can get

$$
\begin{aligned}
& \prod_{j=0}^{i} \varepsilon_{j} \dot{x}_{i}=\sum_{j=0}^{k-1}\left(A_{i j}+A_{i k} P_{j}^{1}\right) x_{j}+A_{i k} y_{k}^{1}, \quad i=\overline{0, k-1}, \\
& \prod_{j=0}^{k} \varepsilon_{j} \dot{y}_{k}^{1}=\left(A_{k k}-\sum_{j=0}^{k-1} \prod_{m=j+1}^{k} \varepsilon_{m} P_{j}^{1} A_{j k}\right) y_{k}^{1} .
\end{aligned}
$$

Consider now the differential expressions

$$
\begin{aligned}
T_{i}^{0}\left(u_{0}^{0}, u_{1}^{0}, \ldots, u_{k-1}^{0}\right)= & \sum_{j=0}^{k-1} \prod_{m=j+1}^{k} \varepsilon_{m}\left(A_{i j}+A_{i k} P_{j}^{1}\right) u_{j}^{0}- \\
& -\prod_{j=1}^{k} \varepsilon_{j} \frac{d}{d t} u_{i}^{0}+A_{i k} y_{k}^{1}, i=\overline{0, k-1} .
\end{aligned}
$$

Let us show, that there exist functions $u_{i}^{0}, i=\overline{0, k-1}$, which can be presented in the form

$$
u_{i}^{0}=\bar{H}_{i}^{1} y_{k}^{1}=\sum_{j=0}^{n} \varepsilon_{k}^{j} H_{i, j}^{1} y_{k}^{1}, \quad i=\overline{0, k-1},
$$

where the matrices $H_{i, j}^{1}, j=\overline{0, n}$, and their $(n-j+1)$ derivatives, are uniformly bounded, and these functions are such that

$$
T_{i}^{0}\left(u_{0}^{0}, u_{1}^{0}, \ldots, u_{k-1}^{0}\right)=o\left(\varepsilon_{k}^{n+1}\right), i=\overline{0, k-1} .
$$


We substitute (3.9) to (3.8) and choose functions $H_{i, j}^{1}, j=\overline{0, n}$, so that in (3.8) all the terms that contain powers of $\varepsilon_{k}$ less than $(n+1)$, are vanishing. For the coefficients $H_{i, j}^{1}$ we obtain

$$
\begin{aligned}
& H_{i, 0}^{1}=A_{i k} A_{k k}^{-1}, \\
& H_{i, j}^{1}=\left(\sum_{m=0}^{k-1} \prod_{l=m+1}^{k-1} \varepsilon_{l} A_{i m} H_{m, j-1}^{1}+\sum_{m=0}^{k-1} \prod_{l=m+1}^{k-1} \varepsilon_{l} \sum_{l=0}^{j-1} H_{i, l}^{1} P_{m, j-1-l}^{1} A_{m k}+\right. \\
& \left.+\sum_{m=0}^{k-1} \prod_{l=m+1}^{k-1} \varepsilon_{l} A_{i k} \sum_{l=0}^{j-1} P_{m, l}^{1} H_{m, j-1-l}^{1}-\prod_{m=0}^{k-1} \varepsilon_{m} \dot{H}_{i, j-1}^{1}\right) A_{k k}^{-1}, \quad j=\overline{1, n} .
\end{aligned}
$$

Now 4) yields that $H_{i, j}^{1}$ and their partial derivatives are bounded. In this case differential expressions (3.8) have the form

$$
T_{i}^{0}\left(u_{0}^{0}, u_{1}^{0}, \ldots, u_{k-1}^{0}\right)=\varepsilon_{k}^{n+1} \mu_{i}^{0}\left(t, \varepsilon_{1}, \varepsilon_{2}, \ldots, \varepsilon_{k}\right) y_{k}^{1},
$$

where $\mu_{i}^{0}, i=\overline{0, k-1}$, are uniformly bounded functions.

If we make the substitution in system (3.7) now

$$
x_{i}=\varepsilon_{k}^{n+1} y_{i}^{1}+\prod_{j=i+1}^{k} \varepsilon_{j} \bar{H}_{i}^{1} y_{k}^{1}, \quad i=\overline{0, k-1},
$$

then for the variables $y_{i}^{1}, i=\overline{0, k}$, we obtain the system

$$
\begin{aligned}
& \prod_{j=0}^{i} \varepsilon_{j} \dot{y}_{i}^{1}=\sum_{j=0}^{k-1}\left(A_{i j}+A_{i k} P_{j}^{1}\right) y_{j}^{1}+\mu_{i}^{0} y_{k}^{1}, \quad i=\overline{0, k-1}, \\
& \prod_{j=0}^{k} \varepsilon_{j} \dot{y}_{k}^{1}=\left(A_{k k}-\sum_{j=0}^{k-1} \prod_{m=j+1}^{k} \varepsilon_{m} P_{j}^{1} A_{j k}\right) y_{k}^{1},
\end{aligned}
$$

of type (2.1), for which there exist integral manifolds

$$
y_{i}^{1}=\prod_{j=i+1}^{k} \varepsilon_{j} H_{i, \star}^{1} y_{k}^{1}, i=\overline{0, k-1} .
$$

If system (3.11) has integral manifolds (3.12), then system (3.7) has integral manifolds

$$
x_{i}=\prod_{j=i+1}^{k}\left(\bar{H}_{i}^{1}+\varepsilon_{k}^{n+1} H_{i, \star}^{1}\right) y_{k}^{1}=\prod_{j=i+1}^{k} H_{i}^{1} y_{k}^{1}, \quad i=\overline{0, k-1},
$$

for which the asymptotic decomposition

$$
x_{i}=\prod_{j=i+1}^{k}\left(\sum_{j=0}^{n} \varepsilon_{k}^{j} H_{i, j}^{1}+\varepsilon_{k}^{n+1} H_{i, \star}^{1}\right) y_{k}^{1}, \quad i=\overline{0, k-1}
$$


is valid.

By making in system (3.7) the substitution

$$
x_{i}=y_{i}^{1}+\prod_{j=i+1}^{k} \varepsilon_{j} H_{i}^{1} y_{k}^{1},
$$

we obtain system (2.4a)-(2.4b) and the first stage of decomposition of (2.1) is finished.

Theorem 2. Let the conditions 1), 2), 4) be true. Then for a sufficiently small $\varepsilon_{k}$ there exists a substitution (2.2), which transforms system (2.1) to form (2.4a)-(2.4b), and the coefficients of the asymptotic decomposition of transformation (2.2) can be uniquely found from algebraic relations (3.3), (3.10).

Consider now $l+1(l \geq 1)$ stage of the decomposition of the system

$$
\begin{gathered}
\prod_{j=0}^{i} \varepsilon_{j} \dot{y}_{i}^{l}=\sum_{j=0}^{k-l} B_{i j}^{l} y_{j}^{l}, \quad i=\overline{0, k-l}, \\
\prod_{j=0}^{i} \varepsilon_{j} \dot{y}_{l}^{k-i+1}=B_{i i}^{k-i+1} y_{i}^{k-i+1}, i=\overline{k-l+1, k} .
\end{gathered}
$$

Assume the following condition to be true:

$5_{l}$ ) matrices $B_{i j}^{l}(i, j=\overline{0, k-l}),\left(B_{k-l, k-l}^{l}\right)^{-1}$ and their $(n+1)$ derivatives are uniformly bounded in $t \in \mathbb{R}$.

Consider the differential expression

$$
T^{l}\left(u^{l}\right)=\sum_{i=0}^{k-l-1} B_{k-l, i}^{l} y_{i}^{l}+B_{k-l, k-l} u^{l}-\prod_{i=0}^{k-l} \varepsilon_{i} \frac{d}{d t} u^{l} .
$$

Let us show that the function $u^{l}\left(t, y_{0}^{l}(t), y_{1}^{l}(t), \ldots, y_{k-l-1}^{l}(t), \varepsilon_{k-l}\right)$, which can be presented in the form

$$
u^{l}=\sum_{i=0}^{k-l-1} \bar{P}_{i}^{l+1}\left(t, \varepsilon_{k-l}\right)=\sum_{i=0}^{k-l-1}\left(\sum_{j=0}^{n} \varepsilon_{k-l}^{j} P_{i, j}^{l+1}(t)\right) y_{i}^{l},
$$

where the matrices $P_{i, j}^{l+1}(t), j=\overline{0, n}, i=\overline{0, k-l-1}$, and their $(n-j+1)$ derivatives are uniformly bounded, is such that for bounded solutions of the system (3.13a) we have

$$
T^{l}\left(u^{l}\right)=o\left(\varepsilon_{k-l}^{n+1}\right)
$$

We substitute (3.15) to (3.14) and choose functions

$$
P_{i, j}^{l+1}(t), j=\overline{0, n}, i=\overline{0, k-l-1},
$$


so that in (3.14) all the terms that contain powers of $\varepsilon_{k-l}$ less than $(n+1)$, are vanishing. For the coefficients in representation (3.2) we obtain

$$
\begin{aligned}
& P_{i, 0}^{l+1}(t)=-\left(B_{k-l, k-l}^{l}\right)^{-1}(t) B_{k-l, i}^{l}(t), \\
& P_{i, j}^{l+1}(t)=\left(B_{k-l, k-l}^{l}\right)^{-1}(t)\left(\prod_{m=1}^{k-l-1} \varepsilon_{m} \dot{P}_{i, j-1}^{l+1}(t)+\sum_{m=0}^{k-l-1} \prod_{s=m+1}^{k-l-1} \varepsilon_{s} P_{m, j-1}^{l+1} B_{m i}^{l}+\right. \\
& \left.+\sum_{m=0}^{k-1-1} \prod_{s=m+1}^{k-l-1} \varepsilon_{s} \sum_{s=0}^{j-1} P_{m, s}^{l+1} B_{m, k-l}^{l} P_{i, j-s-1}^{l+1}\right), \quad j=\overline{1, n}
\end{aligned}
$$

Observe that $\left.5_{l}\right)$ implies that $P_{i, j}^{l+1}(t), j=\overline{0, n}, i=\overline{0, k-l-1}$, and their partial derivatives up to $(n-j+1)$ order, are bounded. If the functions $P_{i, j}^{l+1}$ are chosen by formulaes (3.16), then (3.14) will have the form

$$
T^{l}\left(u^{l}\right)=\varepsilon_{k-l}^{n+1}\left(\sum_{i=0}^{k-l-1} \eta_{i}^{l}\left(t, \varepsilon_{1}, \ldots, \varepsilon_{k-l-1}\right) y_{i}^{l}\right),
$$

where $\eta_{i}^{l}, i=\overline{0, k-l-1}$, are uniformly bounded functions.

If we make in input system (3.13a) the substitution

$$
y_{k-l}^{l}=\sum_{i=0}^{k-l-1} \bar{P}_{i}^{l+1} y_{i}^{l}+\varepsilon_{k-l}^{n+1} z,
$$

then for the variables $y_{0}^{l}, y_{1}^{l}, \ldots, y_{k-l-1}^{l}, z$ we obtain the system

$$
\left\{\begin{aligned}
\prod_{j=0}^{i} \varepsilon_{j} \dot{y}_{i}^{l} & =\sum_{j=0}^{k-l-1}\left(B_{i j}^{l}+B_{i, k-l}^{l} \bar{P}_{j}^{l+1}\right) y_{j}^{l}+\varepsilon_{k-l}^{n+1} B_{i, k-l}^{l} z, \\
i & =\frac{0, k-l-1}{k-l}, \\
\prod_{j=0}^{k-l} \varepsilon_{j} \dot{z} & =\sum_{j=0}^{k-l-1} \eta_{j}^{l}\left(t, \varepsilon_{1}, \ldots, \varepsilon_{k-l-1}\right) y_{j}^{l}+B_{k-l, k-l}^{l} z .
\end{aligned}\right.
$$

System (3.17) is a system of type (3.13a), for which there exists an integral manifold

$$
z=\sum_{i=0}^{k-l-1} P_{i, \star}^{l+1}\left(t, \varepsilon_{k-l}\right) y_{i}^{l}
$$

where $P_{i, \star}^{l+1}, i=\overline{0, k-l-1}$ are uniformly bounded functions.

If system (3.17) has integral manifold (3.18), then system (3.13a) has integral manifold

$$
y_{k-l}^{l}=\sum_{i=0}^{k-l-1}\left(\bar{P}_{i}^{l+1}+\varepsilon_{k-l}^{n+1} P_{i, \star}^{l+1}\right) y_{i}^{l}=\sum_{i=0}^{k-l-1} P_{i}^{l+1} y_{i}^{l},
$$


for which the asymptotic decomposition

$$
y_{k-l}^{l}=\sum_{i=0}^{k-l-1}\left(\sum_{i=0}^{k-l-1} \varepsilon_{k-l}^{j} P_{i, j}^{l+1}+\varepsilon_{k-l}^{n+1} P_{i, \star}^{l+1}\right) y_{i}^{l} .
$$

holds.

By making in system (3.13a) the substitution

$$
y_{k}^{l}=\sum_{i=0}^{k-l-1} P_{i}^{l+1} y_{i}^{l}+y_{k-l}^{l+1},
$$

we can get

$$
\begin{aligned}
& \prod_{j=0}^{i} \varepsilon_{j} \dot{y}_{i}^{l}=\sum_{j=0}^{k-l-1}\left(B_{i j}^{l}+B_{i, k-l}^{l} P_{j}^{l+1}\right) y_{j}^{l}+B_{i, k-l} y_{k-l}^{l+1}, \\
& i=\overline{0, k-l-1}, \\
& \prod_{j=0}^{k-l} \varepsilon_{j} \dot{y}_{k-l}^{l+1}=\left(B_{k-l, k-l}^{l}-\sum_{j=0}^{k-l-1} \prod_{m=j+1}^{k-l} \varepsilon_{m} P_{j}^{l+1} B_{j, k-l}^{l}\right) y_{k-l}^{l+1} .
\end{aligned}
$$

Consider now the differential expressions

$$
\begin{aligned}
T_{i}^{l}\left(u_{0}^{l}, u_{1}^{l}, \ldots, u_{k-l-1}^{l}\right)= & \sum_{j=0}^{k-l-1} \prod_{m=j+1}^{k-l} \varepsilon_{m}\left(B_{i j}^{l}+B_{i, k-l} P_{j}^{l+1}\right) u_{j}^{l}- \\
& -\prod_{j=1}^{k-l} \varepsilon_{j} \frac{d}{d t} u_{i}^{l}+B_{i, k-l} y_{k-l}^{l+1}, i=\overline{0, k-l-1} .
\end{aligned}
$$

Let us show, that there exist functions $u_{i}^{l}, i=\overline{0, k-l-1}$, which can be presented in the form

$$
u_{i}^{l}=\bar{H}_{i}^{l+1} y_{k-l}^{l+1}=\sum_{j=0}^{n} \varepsilon_{k-l}^{j} H_{i, j}^{l+1} y_{k-l}^{l+1}, \quad i=\overline{0, k-l-1},
$$

where matrices $H_{i, j}^{l+1}, j=\overline{0, n}$, and their $(n-j+1)$ derivatives, are uniformly bounded, and these functions satisfy

$$
T_{i}^{l}\left(u_{0}^{l}, u_{1}^{l}, \ldots, u_{k-l-1}^{l}\right)=o\left(\varepsilon_{k-l}^{n+1}\right), i=\overline{0, k-l-1} .
$$

We substitute (3.23) to (3.22) and choose functions $H_{i, j}^{l+1}, j=\overline{0, n}$, so that in (3.22) all the terms that contain powers of $\varepsilon_{k-l}$ less than $(n+1)$, are vanishing. For 
the coefficients $H_{i, j}^{l+1}$ we obtain

$$
\begin{aligned}
& H_{i, 0}^{l+1}=B_{i, k-l}\left(B_{k-l, k-l}^{l}\right)^{-1}, \\
& H_{i, j}^{l+1} \\
& =\left(\sum_{m=0}^{k-l-1} \prod_{s=m+1}^{k-2} \varepsilon_{s} B_{i m}^{l} H_{m, j-1}^{l+1}+\sum_{m=0}^{k-l-1} \prod_{s=m+1}^{k-l-1} \varepsilon_{s} \sum_{s=0}^{j-1} H_{i, s}^{l+1} P_{m, j-s-l}^{l+1} B_{m, k-l}^{l}+\right. \\
& \left.+\sum_{m=0}^{k-l-1} \prod_{s=m+1}^{k-l-1} \varepsilon_{s} B_{i, k-l}^{l} \sum_{s=0}^{j-1} P_{m, s}^{l+1} H_{m, j-1-s}^{l+1}-\prod_{m=0}^{k-l-1} \varepsilon_{m} \dot{H}_{i, j-1}^{l+1}\right)\left(B_{k-l, k-l}^{l}\right)^{-1}, \\
& j=\overline{1, n} .
\end{aligned}
$$

Now $5_{l}$ ) yields that $H_{i, j}^{l+1}$ and their partial derivatives are bounded. In this case differential expressions (3.22) will have the form

$$
T_{i}^{l}\left(u_{0}^{l}, u_{1}^{l}, \ldots, u_{k-l-1}^{l}\right)=\varepsilon_{k-l}^{n+1} \mu_{i}^{l}\left(t, \varepsilon_{1}, \varepsilon_{2}, \ldots, \varepsilon_{k}-l\right) y_{k-l}^{l+1},
$$

where $\mu_{i}^{l}, i=\overline{0, k-l-1}$, are uniformly bounded functions.

If we make the substitution

$$
y_{i}^{l}=\varepsilon_{k-l}^{n+1} y_{i}^{l+1}+\prod_{j=i+1}^{k-l} \varepsilon_{j} \bar{H}_{i}^{l+1} y_{k-l}^{l+1}, \quad i=\overline{0, k-l-1},
$$

to system (3.21) then for variables $y_{i}^{l+1}, i=\overline{0, k-l}$, we obtain the system

$$
\begin{aligned}
& \prod_{j=0}^{i} \varepsilon_{j} \dot{y}_{i}^{l+1}=\sum_{j=0}^{k-l-1}\left(B_{i j}^{l}+B_{i, k-l}^{l} P_{j}^{l+1}\right) y_{j}^{l+1}+\mu_{i}^{l} y_{k-l}^{l+1}, i=\overline{0, k-l-1}, \\
& \prod_{j=0}^{k-l} \varepsilon_{j} \dot{y}_{k-l}^{l+1}=\left(B_{k-l, k-l}^{l}-\sum_{j=0}^{k-l-1} \prod_{m=j+1}^{k-l} \varepsilon_{m} P_{j}^{l+1} B_{j, k-l}\right) y_{k-l}^{l+1}
\end{aligned}
$$

of type (3.13a), for which there exist integral manifolds

$$
y_{i}^{l+1}=\prod_{j=i+1}^{k-l} \varepsilon_{j} H_{i, \star}^{l+1} y_{k-l}^{l+1}, i=\overline{0, k-l-1} .
$$

If system (3.25) has integral manifolds (3.26), then system (3.21) has the integral manifolds

$$
y_{i}^{l}=\prod_{j=i+1}^{k-l}\left(\bar{H}_{i}^{l+1}+\varepsilon_{k-l}^{n+1} H_{i, \star}^{l+1}\right) y_{k-l}^{l+1}=\prod_{j=i+1}^{k-l} H_{i}^{l+1} y_{k-l}^{l+1}, \quad i=\overline{0, k-l-1},
$$


for which the asymptotic decomposition

$$
y_{i}^{l}=\prod_{j=i+1}^{k-l}\left(\sum_{j=0}^{n} \varepsilon_{k-l}^{j} H_{i, j}^{l+1}+\varepsilon_{k-l}^{n+1} H_{i, \star}^{l+1}\right) y_{k-l}^{l+1}, \quad i=\overline{0, k-l-1}
$$

holds.

By making in system (3.21) the substitution

$$
y_{i}^{l}=y_{i}^{l+1}+\prod_{j=i+1}^{k-l} \varepsilon_{j} H_{i}^{l+1} y_{k-l}^{l+1},
$$

we obtain system with $l+1$ decomposed variables and $l+1$ stage of decomposition of the system (2.1) is finished.

Theorem 3. Let the conditions 1), 2) and $\left.5_{l}\right), l=\overline{1, k-1}$, be true. Then for sufficiently small $\varepsilon_{l}$ there exists a substitution (3.20),(3.27), which transforms system (3.13a) to the form

$$
\begin{gathered}
\prod_{j=0}^{i} \varepsilon_{j} \dot{y}_{i}^{l+1}=\sum_{j=0}^{k-l-1} B_{i j}^{l+1} y_{j}^{l+1}, \quad i=\overline{0, k-l-1}, \\
\prod_{j=0}^{k-l} \varepsilon_{j} \dot{y}_{k-l}^{l+1}=B_{k-l, k-l}^{l+1} y_{k-l}^{l+1},
\end{gathered}
$$

and the coefficients of the asymptotic decomposition of the transformation can be uniquely found by (3.16) and (3.24).

Remark 2. By completing $k$ steps of the decomposition of system (2.1) by the scheme described above, we obtain block-diagonal system (2.9). Moreover, the coefficients of the asymptotic decomposition of the transformation can be found by recurrent algebraic relations analogous to (3.16), (3.24).

\section{EXAMPLE.}

Consider the singularly perturbed system of differential equations with constant coefficients and two small parameters

$$
\begin{aligned}
& \dot{x}_{0}=x_{0}+x_{1}+x_{2}, \\
& \varepsilon_{1} \dot{x}_{1}=x_{0}+x_{1}+x_{2}, \\
& \varepsilon_{1} \varepsilon_{2} \dot{x}_{2}=-x_{0}-x_{1}-x_{2} .
\end{aligned}
$$

Let us make the substitution in (4.1) analogous to (2.2)

$$
x_{0}=y_{0}+\varepsilon_{1} \varepsilon_{2} h_{0} w, x_{1}=y_{1}+\varepsilon_{2} h_{1} w, x_{2}=w+p_{0} x_{0}+p_{1} x_{1},
$$


where $p_{0}, p_{1}, h_{0}, h_{1}$ are the solutions of the algebraic systems

$$
\begin{gathered}
\left\{\begin{array}{c}
-1-p_{0}-\varepsilon_{1} \varepsilon_{2} p_{0}-\varepsilon_{1} \varepsilon_{2} p_{0}^{2}-\varepsilon_{2} p_{1}-\varepsilon_{2} p_{0} p_{1}=0, \\
-1-p_{1}-\varepsilon_{1} \varepsilon_{2} p_{0}-\varepsilon_{1} \varepsilon_{2} p_{0} p_{1}-\varepsilon_{2} p_{1}-\varepsilon_{2} p_{1}^{2}=0,
\end{array}\right. \\
\left\{\begin{array}{l}
h_{0}\left(-1+\varepsilon_{1} \varepsilon_{2}+\varepsilon_{2}\right)=1, \\
h_{1}\left(-1+\varepsilon_{1} \varepsilon_{2}+\varepsilon_{2}\right)=1
\end{array}\right.
\end{gathered}
$$

By solving of these systems we obtain

$$
p_{0}=p_{1}=-1, \quad h_{0}=h_{1}=\frac{1}{-1+\varepsilon_{1} \varepsilon_{2}+\varepsilon_{2}} .
$$

Thus the substitution has the form

$$
\begin{gathered}
x_{0}=y_{0}+\frac{\varepsilon_{1} \varepsilon_{2}}{-1+\varepsilon_{1} \varepsilon_{2}+\varepsilon_{2}} w, \quad x_{1}=y_{1}+\frac{\varepsilon_{2}}{-1+\varepsilon_{1} \varepsilon_{2}+\varepsilon_{2}} w, \\
x_{2}=w-x_{0}-x_{1} .
\end{gathered}
$$

In this case system (4.1) at the first step is split to the collection of independent equations

$$
\left\{\begin{array}{l}
\dot{y}_{0}=0, \\
\varepsilon_{1} \dot{y}_{1}=0, \\
\varepsilon_{1} \varepsilon_{2} \dot{w}=\left(-1+\varepsilon_{1} \varepsilon_{2}+\varepsilon_{2}\right) w .
\end{array}\right.
$$

\section{REFERENCES}

[1] E. H. Abed, "Decomposition and stability of multiparameter singular perturbation problems," IEEE Automat. Contr., vol. 31, pp. 925-933, 1986.

[2] S. L. Campbell, Singular systems of differential equations. London: Pitman, 1980.

[3] L.-Y. Chen, N. Goldenfeld, and Y. Oono, "Renormalization group and singular perturbations: Multiple scales, boundary layers, and reductive perturbation theory," Phys. Rev. E, vol. 54, pp. 376-394, 1996, doi: 10.1103/PhysRevE.54.376.

[4] I. M. Cherevko, "Rozsheplennia liniinyh singuliarno zburenyh dyferencial'no-funkcional'nyh rivnian'," Dop. NAN Ukrainy, pp. 32-36, 2002.

[5] I. M. Cherevko and O. V. Osypova, "Rozsheplennia riznotempovyh singuliarno zburenyh liniinyh system," Nauk. visnyk Chernivetskoho universytetu, pp. 78-83, 2012.

[6] M. G. Dmitriev and G. A. Kurina, "Singular perturbations in control problems," Automation and Remote Control, vol. 67, no. 1, pp. 1-43, 2006, doi: 10.1134/S0005117906010012.

[7] V. M. Gol'dshtein and V. A. Sobolev, Kachestvennyj analiz singuliarno vozmushennyh sistem. Novosibirsk, 1988.

[8] D. S. Naidu, "Singular perturbations and time scales in control theory and applications: An overview," Dynamics of Continuous, Discrete and Impulsive Systems, vol. 9, no. 2, pp. 233-278, 2002.

[9] M. M. Semenova, "Dekompozicija sistem s neskol'kimi vremennymi masshtabami," Mehatronika, avtomatizacija, upravlenie, pp. 6-11, 2004

[10] V. A. Sobolev, "Decomposition of linear singularly perturbed systems," Acta Math. Hung., vol. 49, no. 3-4, pp. 365-376, 1987, doi: 10.1007/BF01950998.

[11] V. V. Strygin and V. A. Sobolev, Razdelenie dvizhenij metodom integral'nyh mnogoobrazij. Moscow: Nauka, 1988.

[12] N. V. Voropaeva and V. A. Sobolev, Geometricheskaya dekompozitsiya singulyarno vozmushchennykh sistem. Moscow: Fizmatlit, 2009. 
[13] Y. Zhang, D. S. Naidu, C. Cai, and Y. Zou, "Singular perturbations and time scales in control theory and applications: An overview 2002-2012," International Journal of Information Systems Sciences (IJISS), vol. 9, no. 1, pp. 1-36, 2014.

Authors' addresses

\section{Cherevko}

Yuriy Fedkovych Chernivtsi National University, DMI, 2 Kotsubinsky Str., 58012 Chernivtsi, Ukraine E-mail address: i.cherevko@chnu.edu.ua

\section{O. Osypova}

Yuriy Fedkovych Chernivtsi National University, DMI, 2 Kotsubinsky Str., 58012 Chernivtsi, Ukraine

E-mail address: shurenkacvegmail.com 